\title{
Evaluation of a Web-based Diagnosis Reminder System for Difficult Diagnostic Reasoning
}

\author{
Yasuharu Tokuda $^{1}$, Keijirou Torigoe ${ }^{2}$, Kentaro Matsumoto $^{3}$ and Hideki Yasuda ${ }^{4}$ \\ 1. JCHO Hospitals, Tokyo, 108-8583, Japan \\ 2. Torigoe Clinic, Ibara, Okayama, 715-0025, Japan \\ 3. Osaka National Hospital, Osaka city, Osaka, 540-0006, Japan \\ 4. Yasuda Clinic, Okayama city, Okayama, 700-0862, Japan
}

\begin{abstract}
A web-based diagnosis reminder system may help physicians to perform difficult diagnostic reasoning but its performance has not been evaluated in comparison to that of expert physicians. Clinical case conference was conducted using 10 difficult cases related to internal medicine. Two experienced and expert diagnosticians were invited to comprise a team. A physician assisted with a web-based diagnosis reminder system also participated. Two groups were allowed to propose three possibilities for each case immediately obtaining information on history, physical exam and simple tests. Total scores and the number of accurate diagnoses were greater in the computer-assisted physicianthan in the expert physician team. In conclusion, A web-based diagnosis reminder system can help physicians to well perform difficult diagnostic reasoningcompared to expert physicians.
\end{abstract}

Key words: Computer-aided, decision support, diagnosis reminder, diagnostic error, patient safety.

\section{Introduction}

Diagnostic error has been recognized as an important issue in patient care and innovations have been needed to reduce it. A web-based diagnostic reminder system may be a candidate as an innovative tool. Our system, the Diagnosis Reminder (DR), has been developed as such a diagnostic decision-support tool for physicians in clinics and hospitals (URL: http://60.32.120.74/examples/en/syoujou1.jsp). This web- based system is available to public as open resource and has the English language version. Users can input clinical findings from a patient as key wordsand quickly obtain a list of disease possibilities as about 20-100 diagnoses.

In a previous study [1], its usefulness has been empirically assessed by applying it to the case reports in the New England Journal of Medicine with good results.In the current study, we aimed to prove its usefulness for reminding a physician of a list for

Corresponding author: Yasuharu Tokuda, MD, MPH, research field: general medicine. probable diagnosis in the setting of clinical conference with cases of difficult diagnosis.

\section{Methods}

Clinical conference was organized for diagnosing 10 cases which were selected from the clinical care conundrums cases of the Journal of Hospital Medicine digest book [2]. The cases are related to general internal medicine or hospital medicine. Two physicians (PGY 40 and 13) who had expertise in the diagnosis of general medicine were recruited from local medical community, Kansai area, a southwestern region of Japan, and they were considered as the expert diagnostician team. The DR system was used by author (KT, a physician) of the current study (the computer-assisted physician).

A diagnostic question for each case was asked at the timing immediately after providing history, physical exams, and simple laboratory and imaging test results. The two groups were allowed to provide maximum numbers of three diagnoses with probability ranking (most likely, 2nd likely, 3rd likely) to each case. 
Table 1 Diagnostic possibilities proposed by Expert diagnostician team, Computer-assisted physician, and Computer-generated list of possibilities.

\begin{tabular}{|c|c|c|c|c|}
\hline \multicolumn{5}{|c|}{ Expert diagnostician team } \\
\hline Case No. & Most likely possibility & 2nd likely & 3rd likely & score \\
\hline 1 & Esophageal perforation* & Mediastinitis & Esophageal cancer & 3 \\
\hline 2 & Sezary syndrome & Cytomegalovirus infection & Tuberculosis & 0 \\
\hline 3 & Whipple disease* & Coccidioidomycosis & Amyloidosis & 3 \\
\hline 4 & Hashimoto encephalopathy* & Brainstem encephalitis & Lupus erythematosus & 3 \\
\hline 5 & Thrombotic thrombocytopenic purpura & Toxic shock syndrome* & Evans syndrome & 2 \\
\hline 6 & Constrictive pericarditis* & Amyloidosis & Tuberculosis & 3 \\
\hline 7 & Drug-induced hypersensitivity syndrome & Psoriasis & Yersiniosis & 0 \\
\hline 8 & Crow-Fukase syndrome & Multiple myeloma & IgG4-related disease & 0 \\
\hline 9 & Crow-Fukase syndrome & Multiple myeloma & Amyloidosis & 0 \\
\hline 10 & Amyloidosis & Tuberculosis & Anisakiasis & 0 \\
\hline Total & & & & 14 \\
\hline \multicolumn{5}{|c|}{ Computer-assisted physician } \\
\hline Case No. & Most likely possibility & 2nd likely & 3rd likely & score \\
\hline 1 & Esophageal perforation* & N.P. & N.P. & 3 \\
\hline 2 & Syphilis* & N.P. & N.P. & 3 \\
\hline 3 & Whipple disease* & N.P. & N.P. & 3 \\
\hline 4 & Creuitzfeldt Jacob disease & N.P. & N.P. & 0 \\
\hline 5 & Disseminated intravascular coagulation & Toxic shock syndrome* & Evans syndrome & 2 \\
\hline 6 & Budd Chiari syndrome & Antiphospholipid syndrome & N.P. & 0 \\
\hline 7 & Amyloidosis & Dermatomyositis* & N.P. & 2 \\
\hline 8 & Antiphospholipid syndrome* & N.P. & N.P. & 3 \\
\hline 9 & Sarcoidosis & N.P. & N.P. & 0 \\
\hline 10 & Fulminant Streptococcal infection & N.P. & N.P. & 0 \\
\hline Total & & & & 16 \\
\hline \multicolumn{5}{|c|}{ Computer-generated list of possibilities } \\
\hline Case No. & Most likely possibility & 2nd likely & 3rd likely & score \\
\hline 1 & Acute pericarditis & Empyema & Esophageal perforation* & 1 \\
\hline 2 & Drug-induced disease & Cutanepus lymphoma & Intravascular lymphoma & 0 \\
\hline 3 & Whipple disease* & AIDS & Amyloidosis & 3 \\
\hline 4 & Paraneoplastic syndrome & Drug-induced disease & Refeeding syndrome & 0 \\
\hline 5 & Sepsis & Disseminated intravascular coagulation & Toxic shock syndrome* & 1 \\
\hline 6 & Pulmonary embolism & Amyloidosis & Cardiomyopathy & 0 \\
\hline 7 & Syphilis & Talium intoxication & Lupus erythematosus & 0 \\
\hline 8 & Liver cirrhosis & Antiphospholipid syndrome* & Systemic sclerosis & 2 \\
\hline 9 & Hepatocellular carcinoma* & Insulinoma & Lung cancer & 3 \\
\hline 10 & Gastric cancer & Sepsis & Tuberculosis & 0 \\
\hline Total & & & & 10 \\
\hline
\end{tabular}

*Correct diagnosis. The correct diagnosis of the case 10 was Strongyloidiasis. 
Scoring was conducted as follows: 3 points for making most likely diagnosis correct, 2 points for 2 nd likely, and 1 point for 3rd likely.

\section{Results}

Proposed diagnoses and scoring are shown in Table 1. Total score of the expert diagnostician team was 14 points, while that of the computer-assisted physician was 16 points. The expert diagnostician team provided a total of five correct diagnoses over the 10 cases. The computer-assisted physician provided a total of six correct diagnoses over the 10 cases. Total score of the DR-generated diagnostic list without diagnostic decision by the physician was 10 points. The case number 10 (strongyloidiasis) was not proposed by two groups.

\section{Discussion}

Our results suggest that the DR system could provideuseful information by listing diagnostic possibilities to a physician. In fact, the diagnostic accuracy of the computer-assisted physician was greater than that of the expert diagnostician team. The results of the current study may indicate the preliminary evidence for its usefulness in the setting of clinical conference. However, sole use of the system without decision of a physician performed relatively poorly.

The DR system has disease knowledge database including over 2,000 diseases and 630 clinical manifestations. Our previous report [1] indicated potential usefulness for assisting diagnostic reasoning in cases selected from a major medical journal but a physician assisted with the system had been needed to show good performance comparable to that of expert diagnosticians.

In conclusion, DR systems can be considered useful for assisting good diagnostic reasoning for a physician since it performed better than a team of two expert diagnostician. Further studies are needed for validating its usefulness in diagnosing greater number of cases as well as actual clinical cases.

\section{References}

[1] Torigoe, K., and Tokuda, Y. 2016. "Potential Usefulness of Diagnostic Reminder as Web-based Clinical Decision Support System." Journal of Health Science 4: 297-303. Doi: 10.17265/2328-7136/2016.06.003.

[2] Pile, J. C., Baudendistel, T. E. et al. Clinical Care Conundrums: Challenging Diagnoses in Hospital Medicine (Hospital Medicine: Current Concepts). Wiley-Blackwell, New York, NY. 2013. 